\title{
„Pille“ löst keine Missbildungen aus
}

\author{
Es kommt immer wieder vor, dass Frauen in den drei Monaten vor oder sogar zum Zeitpunkt der Kon- \\ zeption orale Kontrazeptiva nehmen. Es drohen dann aber keine Missbildungen beim Ungeborenen.
}

_ Die dänischen Register verzeichnen zwischen 1997 und 2011 genau 880.694 einzeln lebendgeborene Kinder, die keine Missbildungen bekannter Ursachen wie Alkoholismus der Mutter oder Chromosomendefekte aufwiesen. Dem Verschreibungsregister konnten Daten zu oralen Kontrazeptiva für die Mütter entnommen werden. Unter der Annahme, dass die Frauen die „Pille“ mindestens bis zum Tag der letzten Rezepteinlösung einnahmen, bildete man vier Gruppen: $21 \%$ nahmen nie Kontrazeptiva ein, $69 \%$ bis drei Monate vor der Konzeption, $8 \%$ noch in diesen drei Monaten, $1 \%$ noch nach Schwangerschaftsbeginn.

Insgesamt wurden 22.013 schwere Geburtsdefekte innerhalb des ersten Lebensjahres registriert. In keiner Gruppe war die Entdeckungsrate tendenziell oder gar signifikant verändert. Diese Aussage gilt auch nach Berücksichtigung möglicher Einflussfaktoren wie Alter, Parität, Missbildungen bei Geschwistern, Rauchen, Vorerkrankungen und Medikamenteneinnahme in den letzten sechs Monaten vor der Schwangerschaft.

- Charlton BM, Mølgaard-Nielsen D, Svanström H. Maternal use of oral contraceptives and risk of birth defects in Denmark: prospective, nationwide cohort study. BMJ. 2016;352:h6712

\section{KOMMENTAR \\ Während der Schwangerschaft sollten keine Kontrazeptiva eingenommen werden, doch werden im ersten Jahr etwa 9\% der Frauen trotz Einnahme schwanger. Streng genom- men sollten sogar schon drei Monate vorher keine Kontrazeptiva mehr genommen wer- den, weil sie zu Anstiegen der Vitamin-A- und Abfällen der Folsäure-Konzentration führen können, die für Missbildungen der Kinder}

verantwortlich gemacht werden. Häufig dauert es aber nach Absetzen der Pille nicht drei Monate bis zur Konzeption.

Die Datenlage über die Wirkung von exogenen weiblichen Geschlechtshormonen auf die kindliche Entwicklung ist unzureichend. Einige teils schon ältere Studien haben kindliche Missbildungen bei Einnahme in der Schwangerschaft beschrieben. Das Ergebnis der vorliegenden Studie beruhigt: Orale Kontrazeptiva unmittelbar vor oder während der Schwangerschaft führen nicht zu schweren Missbildungen. Derartige Analysen aus Registern werden häufig gemacht, wenn prospektive Studien nicht möglich sind. Einzelne methodische Ansätze wie der Rückschluss aufMedikamenteneinnahme aus Rezepteinlösungen sind zwar fehleranfällig, aber immerhin besser als retrospektive Befragungen von Probanden.

Prof. Dr. med. H. Holzgreve

\section{Was drückt sich da nach außen?}

Eine 37-jährige Frau suchte die Nothilfe auf wegen einer Vorwölbung im Bereich der linken Flanke (Abb. A). Sie erinnerte sich, dass es kurz nach dem Aufstehen „Plopp“ gemacht hatte, worauf sie die Vorwölbung bemerkt habe. Die Frau war ansonsten gesund, der Befund war nicht druckschmerzhaft. Die differenzialdiagnostischen Überlegungen gingen in Richtung Lipom, Hämatom, Abszess, Fibrom oder maligner Tumor. Erst die CT des Abdomens (Abb. B) erbrachte die richtige, äußerst seltene Diagnose. Man erkannte in der Raumforderung Darmschlingen, sodass die Diagnose einer lumbalen Hernie gestellt werden konnte.

Lumbale Hernien sind ein äußerst seltenes Ereignis. In der Literatur werden nur etwa 300 Fälle berichtet. Sie sind typischerweise in der Region lokalisiert, die von der zwölften Rippe, der Beckenschaufel, dem M. erector trunci und dem M. obliquus externus eingerahmt wird. Etwa $25 \%$ der lumbalen Hernien sind kongenital, ein weiteres Viertel wird durch Trauma oder OP-Narben verursacht, $50 \%$ ereignen sich spontan. Wie bei jeder Hernie findet sich auch hier das typische Phänomen des Hervortretens beim Husten, Niesen und Pressen. Etwa 10\% der Patienten stellen sich wegen einer akuten Inkarzeration vor. Die CT hat den großen Vorteil, dass sie nicht nur die Diagnose sichert, sondern auch Informationen über die Größe des Muskeldefekts und die Muskelschichten, den Hernieninhalt und weitere pathologische Befunde im Bereich des Abdomens liefert. Weil die Hernie in der Regel im Lauf der Jahre größer wird und Inkarzerationen recht häufig sind, wird eine chirurgische Revision bei der Erstvorstellung des Patienten empfohlen.

Prof. Dr. med.H. S. FüeßI

- Falkowitz D, Cambria B, Hahn B. Woman With Mass on Her Back. Ann Emerg Med. 2015;66:684

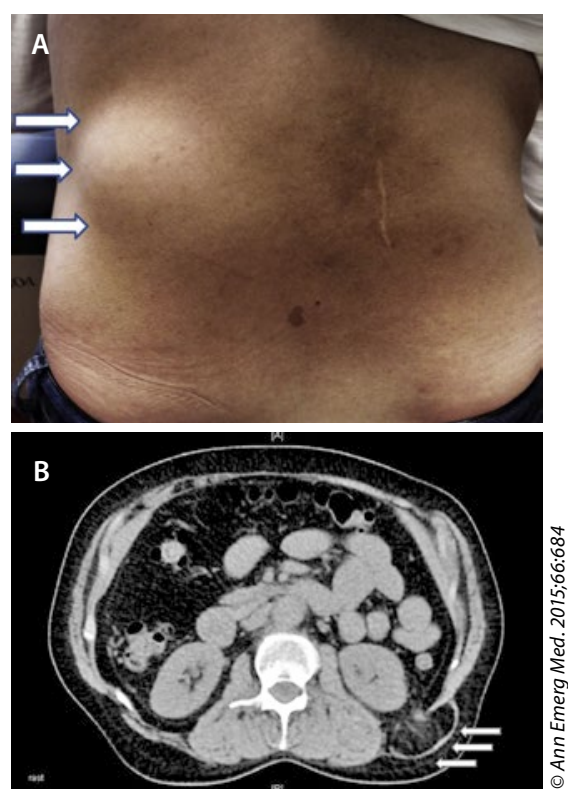

Vorwölbung an der linken Flanke hinten (A, Pfeile); lumbale Hernie im CT des Abdomens (B, Pfeile). 